\title{
Improved care of acute exacerbation of chronic obstructive pulmonary disease in two academic emergency departments
}

\author{
Chu-Lin Tsai • Adit A. Ginde • Phillip G. Blanc • \\ Carlos A. Camargo Jr
}

Received: 2 September 2008 / Accepted: 10 January 2009 / Published online: 14 February 2009

(C) Springer-Verlag London Ltd 2009

\begin{abstract}
Background Although several chronic obstructive pulmonary disease (COPD) practice guidelines have been published, there is sparse data on the actual emergency department (ED) management of acute exacerbation of COPD (AECOPD).

Aims Our objectives were to examine concordance of ED care of AECOPD in older patients with guideline recommendations and to evaluate whether concordance has improved over time in two academic EDs.

Methods Data were obtained from two cohort studies on AECOPD performed in two academic EDs during two different time periods, 2000 and 2005-2006. Both studies included ED patients, aged 55 and older, who presented with AECOPD, and cases were confirmed by emergency physicians. Data on ED management and disposition were obtained from chart review for both cohorts.

Results The analysis included 272 patients: 72 in the 2000 database and 200 in the 2005-2006 database. The mean age of the patients was 72 years; $50 \%$ were women and $80 \%$ white. In 2005-2006, overall concordance with guideline recommendations was high (for chest radiography, pulse oximetry, bronchodilators, all $\geq 90 \%$ ), except for arterial
\end{abstract}

Disclaimer: The views expressed in this paper are those of the author(s) and not those of the editors, editorial board or publisher.

C.-L. Tsai $(\bowtie) \cdot$ P. G. Blanc $\cdot$ C. A. Camargo Jr

EMNet Coordinating Center,

Department of Emergency Medicine,

Massachusetts General Hospital, Harvard Medical School,

326 Cambridge St, Suite 410,

Boston, MA 02114, USA

e-mail: cltsai@post.harvard.edu

\section{A. A. Ginde}

Department of Emergency Medicine,

University of Colorado Denver School of Medicine,

Aurora, CO, USA blood gas testing (7\% among the admitted) and discharge medication with systemic corticosteroids (42\%). Compared to the 2000 data, the use of systemic corticosteroids in the ED improved from 53 to $77 \%$ [absolute improvement: $24 \%, 95 \%$ confidence interval (CI): $11-37 \%$, and the use of antibiotics among the patients with respiratory infection symptoms improved from 56 to $78 \%$ (absolute improvement: 22\%, 95\% CI: 6-38\%).

Conclusions Overall concordance with guidelinerecommended care for AECOPD was high in two academic EDs, and some emergency treatments have improved over time.

Keywords Chronic obstructive pulmonary disease .

Exacerbation · Guidelines · Quality of care

\section{Introduction}

Acute exacerbation of chronic obstructive pulmonary disease (AECOPD) is a common presentation in the emergency department (ED), with an estimated 600,000 ED visits in the USA in 2005 [1]. Despite its importance, research on quality of AECOPD care has received disproportionately less attention. Several COPD practice guidelines are available and provide evidence-based recommendations on AECOPD care; however, the extent to which actual ED practice follows guidelines is largely unknown. We previously showed that concordance of ED management of AECOPD with current treatment guidelines was low at 29 North American EDs in 2000-2001 [2]. Since 2001, there have been several COPD guidelines released, including the Global Initiative for Chronic Obstructive Lung Disease guidelines published in 2001 [3] and updated annually [4], the 2001 American College of 
Physicians guidelines [5], and the 2004 American Thoracic Society and European Respiratory Society joint guidelines [6]. It is unclear whether the publications of these guidelines have helped to improve guideline concordance in AECOPD care over time.

In 2006, we conducted a chart review study on validation of AECOPD administrative codes, which allowed us to reexamine AECOPD care in two of the aforementioned 29 EDs [7]. The two objectives of our study were (1) to investigate recent concordance of ED management of AECOPD in older patients with guideline recommendations in two academic EDs and (2) to evaluate whether the concordance has improved from 2000-2001 to 2005-2006.

\section{Methods}

\section{Study design}

This study combined data from two cohort studies performed during two different time periods, 2000 and 2005-2006. Both were part of the Multicenter Airway Research Collaboration in the Emergency Medicine Network (http:// www.emnet-usa.org).

\section{Study population}

The study conducted in 2006 was a retrospective cohort study using a medical record review in two academic EDs in Boston. Details of the study design and data collection have been published previously [7]. In brief, using the electronic medical records system at each site, we searched the following International Classification of Diseases, Ninth Revision, Clinical Modification (ICD-9-CM) codes to identify possible ED visits for AECOPD from July 2005 to June 2006: 491.2 (obstructive chronic bronchitis), 492.8 (other emphysema), and 496 (chronic airway obstruction, not elsewhere classified) in the primary diagnosis field. We identified all ED visits with candidate ICD-9-CM codes for patients age $\geq 55$ years at each site, selected a random sample of 100 visits from each institution (200 total), and obtained written ED charts. We confirmed AECOPD status by chart review consensus of two emergency physicians (AAG and CAC). Detailed clinical information on the current exacerbation, stable COPD history, ED management, and ED disposition was collected from chart review.

The study conducted in 2000 was a multicenter observational cohort study. To enhance the comparability with the study conducted in 2006, we restricted the multicenter cohort to patients who were enrolled in the two aforementioned EDs in Boston. This would allow us to examine the changes in the care provided by the same EDs over time. Details of the study design and data collection of this multicenter study have been published previously [2]. In brief, investigators at 29 EDs in 15 US states and 3 Canadian provinces provided 24-h/day coverage for a median of 2 weeks. Inclusion criteria were physician diagnosis of COPD; presenting to the ED for treatment of

Table 1 Recommendations for management of acute exacerbation of chronic obstructive pulmonary disease in major clinical guidelines

\begin{tabular}{|c|c|c|c|}
\hline Process of Care & $\begin{array}{l}\text { American College of Chest Physicians \& } \\
\text { American College of Physicians-American } \\
\text { Society of Internal Medicine guidelines } \\
\text { (2001) [5] }\end{array}$ & $\begin{array}{l}\text { American Thoracic Society/ } \\
\text { European Respiratory Society } \\
\text { guidelines (updated in 2004) } \\
\text { [6] }\end{array}$ & $\begin{array}{l}\text { Global Initiative for Chronic } \\
\text { Obstructive Lung Disease (updated } \\
\text { in 2007) [4], strength of evidence } \\
\text { where indicated }\end{array}$ \\
\hline \multicolumn{4}{|l|}{ Diagnostic evaluation } \\
\hline Chest radiography & Recommended & Recommended in admitted patients & Recommended \\
\hline Pulse oximetry & Recommended & Recommended & Recommended \\
\hline Arterial blood gas & Not clearly addressed & Recommended in admitted patients & Recommended in admitted patients \\
\hline Acute spirometry & Not recommended & Not addressed & Not recommended ${ }^{\mathrm{a}}$ \\
\hline \multicolumn{4}{|l|}{ ED treatment } \\
\hline Inhaled bronchodilators & Recommended & Recommended & Recommended (level A) \\
\hline Systemic corticosteroids & Recommended & Recommended $^{\mathrm{b}}$ & Recommended (level A) \\
\hline Antibiotics & Recommended in severe exacerbations & $\begin{array}{l}\text { Recommended in patients with altered } \\
\text { sputum purulence and/or volume }\end{array}$ & $\begin{array}{l}\text { Recommended in patients with airway } \\
\text { infection (level B) }\end{array}$ \\
\hline Methylxanthines & Not recommended & Not addressed ${ }^{c}$ & Second-line therapy (level B) ${ }^{\mathrm{d}}$ \\
\hline \multicolumn{4}{|l|}{ Discharge medication } \\
\hline Systemic corticosteroids & Recommended & Recommended & Recommended (level A) \\
\hline
\end{tabular}

$E D$ emergency department, level $A$ randomized controlled trials with rich body of data, level $B$ randomized controlled trials with limited body of data

${ }^{a}$ Changed from "not clearly addressed" in 2001 guidelines

${ }^{\mathrm{b}}$ Changed from "may be added" in 1995 guidelines

${ }^{\mathrm{c}}$ Changed from "primary agent" in 1995 guidelines

"Changed from "level A recommended treatment" in 2001 guidelines 
AECOPD, as defined by increasing shortness of breath, worsening cough, or change in sputum production [4, 6]; age $\geq 55$ years; and the ability to give informed consent. Patients' demographics, stable COPD history, and details of their current AECOPD were obtained by ED interview. Data on ED management and disposition were obtained using medical chart review. For those who did not complete ED interview (missed by investigators, refused, or other reasons), chart review was performed to abstract complete information on demographics and ED management, along with limited information on stable COPD history and current exacerbation.

For the chart review in both studies, trained chart abstractors used a standardized form with precisely predefined variables to collect data. Periodic meetings were held to resolve coding issues and to monitor data collection. Chart reviewers were blinded to the study hypothesis. Repeat visits by individual subjects were excluded. The Institutional Review Boards approved the studies.
Outcome measures

\section{Concordance with guidelines: quality indicators}

On the basis of recommendations contained in the COPD guidelines [4-6], we categorized the following diagnostic evaluations and treatments in the ED as beneficial: chest radiography, pulse oximetry, arterial blood gas analysis, inhaled bronchodilators (either short-acting beta-agonists or inhaled anticholinergics), systemic corticosteroids, antibiotics, and discharge medications with systemic corticosteroids (Table 1). Bronchodilator and systemic corticosteroid treatments were recently included as quality measures in pharmacotherapy for AECOPD in the Health Care Effectiveness Data and Information Sets [8]. To better define the patient population eligible for the quality indicators, we restricted the denominator of arterial blood gas analysis to admitted patients as appropriate candidates (i.e., severe

Table 2 Clinical characteristics and quality of care received among emergency department patients with an acute exacerbation of chronic obstructive pulmonary disease, according to time period

\begin{tabular}{|c|c|c|c|}
\hline Variables & Period 1: $2000(n=72)$ & Period 2: 2005-2006 $(n=200)$ & Difference in means or proportions $(95 \% \mathrm{CI})$ \\
\hline \multicolumn{4}{|l|}{ Baseline clinical characteristic } \\
\hline Age, years & $71 \pm 9$ & $72 \pm 9$ & $1(-2$ to 3$)$ \\
\hline Female sex & $36 / 72(50)$ & $101 / 200(51)$ & $1(-13$ to 14$)$ \\
\hline White & $58 / 68(85)$ & $156 / 200(78)$ & $-7(-17$ to 3$)$ \\
\hline Current or former smoker & 68/71 (96) & $154 / 183(84)$ & $-12(-5$ to -19$)$ \\
\hline Home oxygen use & $15 / 47(32)$ & $72 / 200(36)$ & $4(-11$ to 19$)$ \\
\hline \multicolumn{4}{|l|}{ Acute exacerbation } \\
\hline Duration of symptoms $\geq 24 \mathrm{~h}$ & $56 / 69(81)$ & $138 / 164(84)$ & $3(-8$ to 14$)$ \\
\hline Triggered by respiratory infection & $45 / 69(65)$ & $116 / 196(59)$ & $-6(-19$ to 7$)$ \\
\hline Respiratory rate, breaths/min & $26 \pm 7$ & $23 \pm 6$ & $3(1$ to 5$)$ \\
\hline $\mathrm{O}_{2}$ saturation on room air, $\%{ }^{\mathrm{a}}$ & $89 \pm 10$ & $92 \pm 6$ & $4(1$ to 7$)$ \\
\hline \multicolumn{4}{|l|}{ Process of care } \\
\hline \multicolumn{4}{|l|}{ Recommended } \\
\hline Chest radiography & $72 / 72(100)$ & 196/200 (98) & $-2(-5$ to 3$)$ \\
\hline Pulse oximetry & $72 / 72(100)$ & $199 / 200(99.5)$ & $-0.5(-3$ to 5$)$ \\
\hline Arterial blood gas ${ }^{\mathrm{b}}$ & $14 / 58(24)$ & $12 / 166(7)$ & $-17(-5$ to -27$)$ \\
\hline Received inhaled bronchodilators in ED & $59 / 71(83)$ & $180 / 200(90)$ & $7(-3$ to 17$)$ \\
\hline Received systemic corticosteroids in ED & $38 / 72(53)$ & $154 / 200(77)$ & 24 (11 to 37$)$ \\
\hline Received antibiotics in $\mathrm{ED}^{\mathrm{c}}$ & $25 / 45(56)$ & $90 / 116(78)$ & $22(6$ to 38$)$ \\
\hline Sent home on systemic corticosteroids ${ }^{\mathrm{d}}$ & $6 / 11(55)$ & $13 / 31(42)$ & $-13(-22$ to 47$)$ \\
\hline \multicolumn{4}{|c|}{ Not recommended } \\
\hline Spirometry ${ }^{\mathrm{e}}$ & $7 / 35(20)$ & $19 / 200(10)$ & $-11(-24$ to 3$)$ \\
\hline Received methylxanthines in ED & 0 & 0 & 0 \\
\hline \multicolumn{4}{|l|}{ ED disposition } \\
\hline Hospital admission $^{\mathrm{f}}$ & $58 / 72(81)$ & $166 / 200(83)$ & $2(-8$ to 13$)$ \\
\hline
\end{tabular}

$E D$ emergency department

Data are presented as number/total number (percentage) or mean \pm standard deviation, unless otherwise specified. For certain variables, the sample size may vary because of missing data and/or restrictions to the denominators

${ }^{a}$ Restricted to room air oxygen saturations ( $n=53$ for period $1, n=49$ for period 2$)$

${ }^{\mathrm{b}}$ Restricted to admitted patients

${ }^{\mathrm{c}}$ Restricted to patients with respiratory infection symptoms

${ }^{\mathrm{d}}$ Restricted to patients sent home from ED

${ }^{\mathrm{e}}$ Excluded patients enrolled in a substudy of acute spirometry in the 2000 cohort to reflect normal clinical practice

${ }^{\mathrm{f}}$ Hospital admission was defined as admission to an inpatient unit, observation unit, or intensive care unit 
exacerbation) [9]. We also restricted the denominator of antibiotic use to patients with respiratory infection symptoms, according to guideline recommendations [4-6]. Finally, we considered evaluation by acute spirometry and treatment with methylxanthine bronchodilators as not recommended [4-6].

\section{Statistical analysis}

Summary statistics are presented as proportions [with $95 \%$ confidence intervals $(\mathrm{CI})$ ] or means [with standard deviations (SD)]. The univariable associations between time period and variables were examined using two-sample $t$-tests or tests of proportions, as appropriate. To adjust for baseline patient characteristics (patient mix) that may have confounded the relationship between time period and quality indicators, multivariable logistic regression modeling was performed. Model variables included age, sex, race, duration of symptoms, respiratory infection triggers, respiratory rate, oxygen saturation on room air, and indicators for the two time periods. Variables with missing data were dummy coded using the missing-indicator method. All odds ratios (OR) are presented with $95 \%$ CIs. All analyses were performed using Stata v10.0 software (StataCorp, College Station, TX, USA). All $P$ values are two-sided, with $P<0.05$ considered statistically significant.

\section{Results}

Overall, 272 patients were included in the current analysis. The mean age of these patients was 72 years (SD: 9); $50 \%$ were women and $80 \%$ were white. There were 72 patients with AECOPD in the 2000 database and 200 in the 20052006 database. Of the 72 patients in 2000, 46 underwent interview plus chart review and 26 underwent chart review only. There were no significant differences in age, sex, or race between the two cohorts (Table 2). Compared with patients in the earlier study, patients in 2005-2006 were less likely to be current or former smokers and presented to the ED with lower acuity, as suggested by the lower respiratory rates and the higher oxygen saturation on room air. There were no significant differences in home oxygen use, duration of symptoms, or respiratory infection triggers between the two cohorts.

With respect to quality of emergency care, the use of chest radiography, pulse oximetry, and bronchodilators was consistently high during the study period (Table 2 and Fig. 1). In contrast, the use of arterial blood gas testing among the admitted decreased significantly from 24 to $7 \%$ (absolute change: $-17 \%, 95 \%$ CI: -5 to $-27 \%$ ). The use of systemic corticosteroids in the ED increased significantly from $53 \%$ in 2000 to $77 \%$ in $2005-2006$ (absolute improvement: $24 \%$, 95\% CI: 11-37\%). Likewise, the use of antibiotics among the

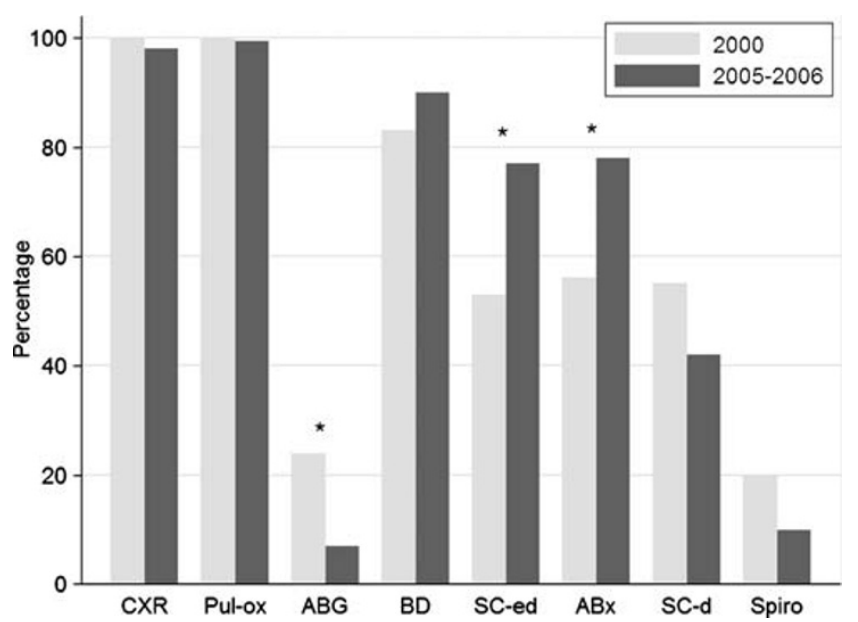

Fig. 1 Absolute change in performance on quality indicators for acute exacerbation of chronic obstructive pulmonary disease among eligible patients in the emergency departments. Asterisks indicate $P<0.05$. $C X R$ chest X-ray, Pul-ox pulse oximetry, $A B G$ arterial blood gas, $B D$ bronchodilators, $S C$-ed systemic corticosteroids in the emergency department, $A B x$ antibiotics in the emergency department, $S C$-d systemic corticosteroids at discharge, Spiro spirometry

patients with respiratory infection symptoms improved from 56 to $78 \%$ (absolute improvement: $22 \%, 95 \%$ CI: 6-38\%). The prescription of systemic corticosteroids at discharge, however, was not high in either time period $(<60 \%)$, and no significant changes were observed during the study period.

With respect to ED management that is not guideline recommended, there was a decreasing trend in the use of acute spirometry, but this was not statistically significant. Use of methylxanthines was not observed during the study period. More than $80 \%$ of the patients were admitted, with this high admission rate persisting during the study period. The pattern of changes in the performance on quality indicators remained after adjusting for small baseline differences in patient characteristics between the two time periods in the logistic regression models. For example, the increasing trends in the use of systemic corticosteroids and antibiotics in the ED persisted (adjusted OR for corticosteroid use comparing 2005-2006 to 2000: 3.4, 95\% CI: $1.7-7.0$; adjusted OR for antibiotic use: $4.3,95 \%$ CI: $1.5-12.2)$.

\section{Discussion}

These data demonstrate overall high concordance of ED management of AECOPD in older patients with guideline recommendations in two academic EDs in Boston, except for arterial blood gas testing and discharge medication with systemic corticosteroids. In addition, we found significant improvements in some guideline-recommended treatments, including an increase in the ED use of systemic corticosteroids and antibiotics. 
Several guideline-recommended processes of care appeared to have reached the "ceiling" effect in the two academic EDs, including the use of chest radiography, pulse oximetry, and bronchodilators. On the other hand, the non-use of methylxanthines and rare use of acute spirometry appeared to have reached the "floor" effect, which also is encouraging. Spirometry is often difficult to perform in the emergency setting, and its accuracy is questionable $[4,5]$. Therefore acute spirometry should have a very limited role in AECOPD [10].

The observed improvements in prescriptions of systemic corticosteroids and antibiotics for AECOPD are consistent with the national trends [1]. Furthermore, the prescription rates of the medications in the two academic EDs were better than the corresponding national averages in 2005 (national averages: $60 \%$ for systemic corticosteroids and $42 \%$ for antibiotics) [1]. This may suggest that the diffusion of the guideline-recommended care has been relatively successful in these two academic EDs. Continuous quality improvement efforts, such as educational activities, should help shift the average performance toward excellence.

A finding that is discordant with guideline recommendations was the low use of arterial blood gas analysis; however, this may reflect actual practice. Given that oxygenation can be assessed by pulse oximetry and that ventilation can be monitored by noninvasive capnography [10], the low use of arterial blood gas analysis might be acceptable. Alternatively, patients might receive this procedure after their admission decisions were made (i.e., as inpatients). This also might contribute to the low use of this procedure in the ED. The low prescription of systemic corticosteroids at ED discharge also is somewhat concerning, a finding that needs to be confirmed in future studies with more discharged patients.

This study has some potential limitations. First, the guideline-concordant care is only reflective of emergency care processes at two well-established teaching hospitals and may not be fully generalizable to community hospitals. Second, the data on quality of care were obtained using medical record review for both cohorts, and some of the quality deficit may be due to underdocumentation. However, previous studies of acute asthma showed that the rates of ED assessments and treatments by chart abstraction were similar to those by direct observation, with kappa coefficients ranging from 0.6 to 0.9 [11]. In addition, such underdocumentation would make it difficult to explain both the increasing and the decreasing trends that we identified during the study period. Finally, there may be a Hawthorne effect created by the patient enrollment process in the 2000 study (i.e., short-term improvement due to observation). However, had this effect been removed, the improving trend we observed would have been more significant.
In summary, we found overall high concordance of ED management of AECOPD with guideline recommendations in two Boston academic EDs, with significant improvements in some treatments between 2000 and 2006. In this small sample of academic EDs, there remain opportunities for improvement. Continuous quality improvement efforts in refinement, dissemination, and implementation of clinical practice guidelines may further improve quality of AECOPD care. Future COPD guidelines should consider incorporating these data to better reflect the ED aspect of AECOPD care.

Funding This project was supported by a grant from National Committee for Quality Assurance (Washington, DC).

Conflicts of interest Dr. Camargo has received financial support (research grants, consulting, lectures) in the past 5 years from Altana, AstraZeneca, Aventis, Aventis Pasteur, Boehringer Ingelheim, Dey, GlaxoSmithKline, MedImmune, Merck, Novartis, Pfizer, Respironics, and Schering Plough. Other authors have no conflicts of interest to disclose.

\section{References}

1. Tsai CL, Sobrino JA, Camargo CA Jr (2008) National Study of Emergency Department Visits for Acute Exacerbation of Chronic Obstructive Pulmonary Disease, 1993-2005. Acad Emerg Med 15:1275-1283

2. Cydulka RK, Rowe BH, Clark S, Emerman CL, Camargo CA Jr (2003) Emergency department management of acute exacerbations of chronic obstructive pulmonary disease in the elderly: the Multicenter Airway Research Collaboration. J Am Geriatr Soc 51 (7):908-916

3. Pauwels RA, Buist AS, Calverley PM, Jenkins CR, Hurd SS, GOLD Scientific Committee (2001) Global strategy for the diagnosis, management, and prevention of chronic obstructive pulmonary disease. NHLBI/WHO Global Initiative for Chronic Obstructive Lung Disease (GOLD) Workshop summary. Am J Respir Crit Care Med 163(5):1256-1276

4. Global Initiative for Chronic Obstructive Lung Disease (GOLD) (2007). NHLBI/WHO Global Strategy for the Diagnosis, Management and Prevention of COPD. Available via: http://www.goldcopd.org.

5. Bach PB, Brown C, Gelfand SE, McCrory DC, American College of Physicians-American Society of Internal Medicine, American College of Chest Physicians (2001) Management of acute exacerbations of chronic obstructive pulmonary disease: a summary and appraisal of published evidence. Ann Intern Med 134(7):600-620

6. Celli BR, MacNee W, ATS/ERS Task Force (2004) Standards for the diagnosis and treatment of patients with COPD: a summary of the ATS/ERS position paper. Eur Respir J 23(6):932-946

7. Ginde AA, Tsai CL, Blanc P, Camargo CA Jr (2008) Positive predictive value of ICD-9-CM codes to detect acute exacerbation of COPD in the emergency department. Jt Comm J Qual Patient Saf 34:678-680

8. National Committee on Quality Assurance (2008) The Health Care Effectiveness Data and Information Sets (HEDIS), 2008 Quality Measures for Pharmacotherapy of COPD Exacerbation. 
Available via: http://www.ncqa.org/Portals/0/HEDISQM/ HEDIS2008/2008 Measures.pdf. Accessed July 10

9. Rodriguez-Roisin R (2000) Toward a consensus definition for COPD exacerbations. Chest 117(5 Suppl 2):398S-401S

10. Smithline HA, Rowe BH, Radeos MS, Cydulka RK, Camargo CA Jr, Working Group on Emergency Management of COPD Exacerbations (2005) Research opportunities in the management of acute exacerbations of chronic obstructive pulmonary disease. Acad Emerg Med 12(8):742-750

11. McDermott MF, Lenhardt RO, Catrambone CD, Walter J, Weiss KB (2006) Adequacy of medical chart review to characterize emergency care for asthma: findings from the Illinois Emergency Department Asthma Collaborative. Acad Emerg Med 13(3):345348 